\title{
Computation of the Short-Time Linear Canonical Transform with Dual Window
}

\author{
Lei Huang, Ke Zhang, Yi Chai, and Shuiqing Xu \\ State Key Laboratory of Power Transmission Equipment and System Security and New Technology, College of Automation, \\ Chongqing University, Chongqing 400044, China \\ Correspondence should be addressed to Ke Zhang; zhangkecqu@yeah.net
}

Received 25 April 2017; Revised 19 July 2017; Accepted 1 August 2017; Published 26 September 2017

Academic Editor: Aimé Lay-Ekuakille

Copyright (C) 2017 Lei Huang et al. This is an open access article distributed under the Creative Commons Attribution License, which permits unrestricted use, distribution, and reproduction in any medium, provided the original work is properly cited.

\begin{abstract}
The short-time linear canonical transform (STLCT), which maps the time domain signal into the joint time and frequency domain, has recently attracted some attention in the area of signal processing. However, its applications are still limited due to the fact that selection of coefficients of the short-time linear canonical series (STLCS) is not unique, because time and frequency elementary functions (together known as basis function) of STLCS do not constitute an orthogonal basis. To solve this problem, this paper investigates a dual window solution. First, the nonorthogonal problem that suffered from original window is fulfilled by orthogonal condition with dual window. Then based on the obtained condition, a dual window computation approach of the GT is extended to the STLCS. In addition, simulations verify the validity of the proposed condition and solutions. Furthermore, some possible applied directions are discussed.
\end{abstract}

\section{Introduction}

The short-time Fourier transform (STFT) is proved to be a potent tool for processing nonstationary signals [1]. It can map the time domain signal into the joint time and frequency domain by adding a window to the Fourier transform (FT). When we select Gaussian window, the STFT becomes the Gabor transform (GT). In recent years, with the development of nonstationary signal processing technology, the linear canonical transform (LCT) was developed by many scholars [2-9]. It is a generalized form of the FT and the Fractional Fourier transform (FRFT) and has been considered to be a powerful analyzing tool in signal processing and optics [10$15]$.

Serving as a powerful analyzing tool, basic theories of the LCT are fruitful $[12,16-19]$. But it fails in locating the LCT-frequency contents due to its global kernel. Kou and Xu, in [20], proposed the short-time linear canonical transform (STLCT) to handle that limitation by adding a window to the LCT. Subsequently, the STLCT also received attention from some scholars. Some theoretical results in the STFT domain have been extended to the STLCT domain. In [21], Kou et al. proved the Paley-Wiener theorems for the STLCT in mathematical applications. In [22], Zhang addressed a sampling theorem for the STLCT by means of a generalized Zak transform associated with the LCT. In [23], Guanlei discussed the uncertainty relation in two STLCT domains.

In our previous work [24], we studied the problem of uncertainty principle and general orthogonal condition of the STLCT by generalizing the uncertainty principle and orthogonal condition of the STFT to the STLCT. However, its applications are still limited due to the fact that selection of coefficients of the short-time linear canonical series (STLCS) is not unique. In this paper, we study the problem of computation of the STLCS. Our object is to find an appropriate improved orthogonal condition and focus on the computation method that can be applied to the STLCS.

Computation of coefficients of the STLCS depends on orthogonalization of its basis composed of selected window and kernel function. In fact, computation of the GT, special case of the STLCT, was once limited due to the difficulties in computing the Gabor series (GS) coefficients because of its nonorthogonal basis (see Figure 1). One way to address this is by introducing orthogonal condition, which was first proposed by Bastiaans in 1980 [25]. Based on Bastiaans' work, the dual window solution of the GS was then founded in 


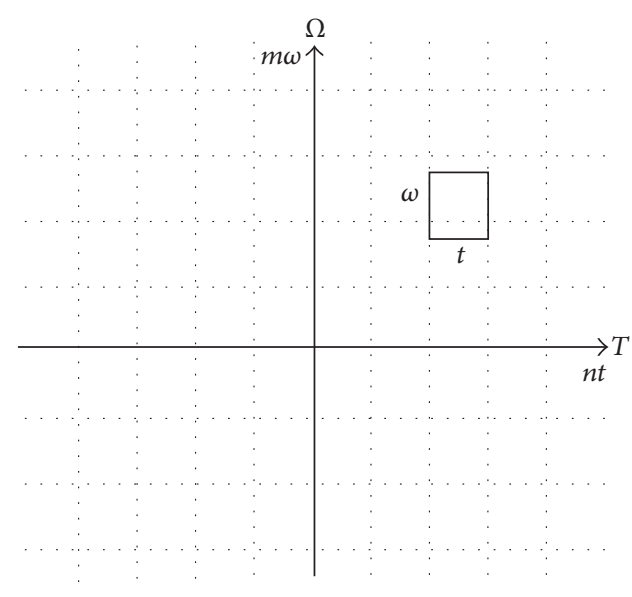

(a)

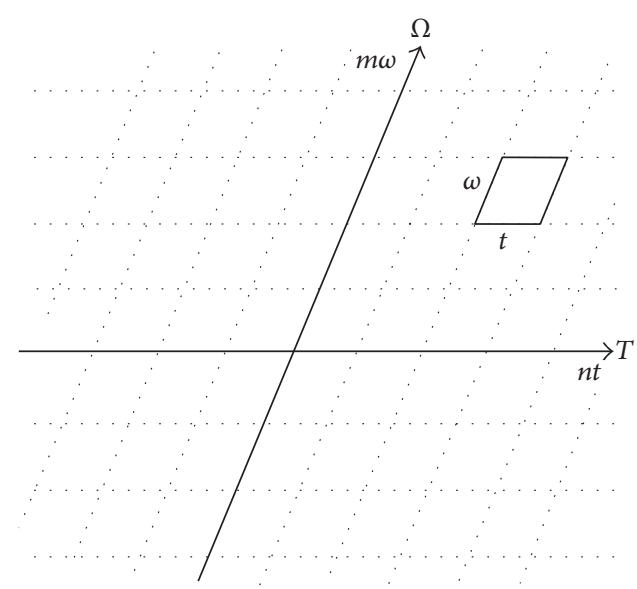

(b)

FIGURE 1: The comparison of time-frequency plane. (a) Gabor series plane; (b) STLCT series plane.

[26-28]. All these computing methods of dual window are determined by the orthogonal condition and its equivalent form or their corollaries. It is natural to solve nonorthogonal problem of the STLCS by extending the solutions of the GS.

The difficulties of generalizing these approaches are as follows: the first one is how to obtain an appropriate equivalent form of orthogonal condition of the STLCS that can be used in subsequent dual window computation; second challenge is how to use this condition to compute dual window functions. As mentioned above, there were several ways to compute the dual window of the GS, including frame theorem [27], orthogonal analysis [28], and minimum energy approach [26]. Each approach had different requirement of orthogonal condition. For instance, the methods in $[27,28]$ needed biorthogonal condition, a stronger orthogonal condition, to compute the dual window. Compared to the frame method and biorthogonal analysis, the approach in [26] was based on a normal orthogonal condition and avoids computing frame bounds. But the process needed an appropriate corollary of the condition to accomplish the derivation process, and the calculation is simple.

In this paper, in order to employ and extend the classic GS minimum energy approach, we seek an appropriate orthogonal condition of the STLCS based on the previous work $[24,25]$, which can be viewed as existence condition of the dual window. Two specific forms of orthogonal condition that meet the needs of subsequent dual window computation process are obtained. Then GS minimum energy approach is extended to the STLCS. To preserve the fine properties of original window, the dual window should be as similar as possible to the "shape" of the original Gaussian window, which can be achieved by adjusting free parameters.

The rest of this paper is organized as follows. In Section 2, the LCT and STLCS are briefly introduced. In Section 3, two specific forms of orthogonal condition known as existence condition of dual window function for STLCS are derived. In Section 4, we extend a minimum energy approach to compute dual window function by given original window.
Simulation and discussion are also provided in this part. Section 5 concludes this paper.

\section{Preliminaries}

2.1. Review of the LCT and LCS. The linear canonical transform (LCT), a generalization of the Fourier transform (FT) and the fractional Fourier transform (FRFT), is a very useful signal analysis tool. It is an integral transform with four parameters $A=(a, b, c, d)$. It is defined as follows [5]:

$$
L_{A}(f)(u)=\left\{\begin{array}{l}
\int_{-\infty}^{\infty} O_{A}(t, u) f(t) d t, b \neq 0 \\
\sqrt{d} e^{j(c d / 2) u^{2}} f(d u), b=0,
\end{array}\right.
$$

where $a, b, c, d \in \mathbb{R}$ and $a d-b c=1$. And the kernel of LCT is

$$
O_{A}(t, u)=\frac{1}{\sqrt{j 2 \pi b}} e^{(j / 2 b)\left(a t^{2}-2 t u+d u^{2}\right)} \quad b \neq 0
$$

when $(a, b, c, d)=(0,1,-1,0)$, and the LCT reduces to the multiplied FT:

$$
\operatorname{LCT}_{(0,1,-1,0)}(f(t))(u)=\sqrt{-j} \operatorname{FT}(f(t))(u)
$$

when $(a, b, c, d)=(\cos \alpha, \sin \alpha,-\sin \alpha, \cos \alpha)$, and it reduces to the FRFT multiplied by a constant phase:

$$
\begin{gathered}
\operatorname{LCT}_{(\cos \alpha, \sin \alpha,-\sin \alpha, \cos \alpha)}(f(t))(u) \\
=\sqrt{e^{-j \alpha}} \operatorname{FRFT}(f(t))(u) .
\end{gathered}
$$

The LCT can also be extended to include the Fresnel transform and Laplace transform. Besides, as its series form, the linear canonical series (LCS) is an another expression 
form of the LCT from the perspective of theory of series. Its basis function is defined as follows [12]:

$$
\begin{aligned}
& O_{A, n}(t) \\
& \quad=\frac{1}{\sqrt{-j 2 \pi b}} \exp \left\{-\frac{j\left[a t^{2}+d\left(n u_{0}\right)^{2}\right]}{2 b}+\frac{j\left(n u_{0}\right) t}{b}\right\},
\end{aligned}
$$

where $u_{0}$ is called the central frequency. The basis of LCS is orthogonal with an aperiodic chirp function. For more details about the definition, properties, and relationship between the LCT and LCS, see [12].

2.2. The STLCT and STLCS. The LCT is a potent tool to analyze signal. But it cannot reveal the local frequency contents because of its global kernel. To tackle this problem, by adding a suitable window to the LCT, it is defined as follows [20]:

$$
\operatorname{STLCT}_{f, A}(t, u)=\int_{-\infty}^{+\infty} f(\tau) g(\tau-t) O_{A}(\tau, u) d \tau .
$$

By definition, the result of the integral transform contains information about time $t$ and frequency $u$. So we can know not only the STLCT frequency domain contents but also how they change with time. The basis of the STLCT is denoted by

$$
y(\tau \mid t, u)=g(\tau-t) O_{A^{-1}}(t, u) .
$$

Based on the expanding theory of Gabor series, a real signal can be expanded on the Gabor basis under certain conditions. As the generalized form of Gabor series, a real signal can also be expressed by STLCS. It can be defined as the following form:

$$
f(t)=\sum_{m} \sum_{n} C_{A, m, n} h_{A, m, n}(t)
$$

The expansion coefficients $C_{A, m, n}$ are the sampled values of STLCT. Let $u_{0}=\omega b$ and substitute it into (5); then the basis of the STLCS can be obtained as follows:

$$
\begin{aligned}
& h_{A, m, n}(t)=h(t-m T) \\
& \cdot \exp \left\{j\left[-\frac{1}{2}\left(\frac{a}{b} t^{2}+\frac{d}{b}(n \omega b)^{2}\right)+n t \omega\right]\right\},
\end{aligned}
$$

where $m, n \in \mathbb{Z}, T$ is the time translation parameter, and the number $\omega$ is called the frequency modulation parameter.

\section{Orthogonal Condition of the STLCS}

To solve the nonorthogonal basis problem of the Gabor, Bastiaans proposed a biorthogonal condition of the GS to disclose orthogonal relationship between original and dual window, which was given as

$$
\begin{aligned}
& \frac{T_{0} \Omega_{0}}{2 \pi} \int_{-\infty}^{+\infty} h(t) \gamma^{*}\left(t-m T_{0}\right) \exp \left(-j n \Omega_{0} t\right) d t \\
& \quad=\delta(m) \delta(n),
\end{aligned}
$$

where $T_{0}$ and $\Omega_{0}$ represent time and frequency sampling intervals, respectively. The equation denotes the relationship between dual window $\gamma(t)$ and original window $h(t)$, in which $\gamma^{*}(\cdot)$ is conjugate form of $\gamma(\cdot)$. And the dual window $\gamma(t)$ existing in the above formula guarantees the completeness of the expansion coefficients.

But above all, the GS is the special case of the STLCS and also faced its nonorthogonal basis problem. Our object is to obtain an appropriate equivalent form of orthogonal condition of the STLCS which can be used in dual window calculation. In [22], Zhang proposed a biorthogonal condition for the STLCS, which can be viewed as a theoretical foundation for frame theorem approach and orthogonal analysis method in seeking dual window. However, the process is too complex such as frame boundary estimation and matrix calculation. So the classic GS minimum energy approach is extended to the dual window computation via an orthogonal condition, a simpler condition. And in our previous work [24], we proposed an orthogonal condition for the STLCS by extending Bastiaans' work in [25].

In this section, based on the work in Theorem 2, [24], we find two specific orthogonal conditions so as to apply to subsequent dual window deducing. The main result of this section is summarized as the following theorem.

Theorem 1. The basis of the STLCS is of orthogonality if and only if there exists a dual window function $\gamma(t)$ satisfying the following orthogonal condition:

$$
\begin{aligned}
T_{0} \sum_{n} h^{*}(t-n T) \gamma(t-n T) & =1 \\
\sum_{n} \gamma(t-n T) h^{*}\left(t-n T-m T_{0}\right) & =0,
\end{aligned}
$$

where $T_{0}=b / \omega$ and $T$ denote the time and frequency sampling steps, respectively. $\gamma^{*}(\cdot)$ is conjugate form of $\gamma(\cdot)$.

Proof. Suppose there exists a dual window function that can meet the need of orthogonality, and the dual window constitutes a dual basis, such that

$$
\left\langle h_{A, m, n}(t), \gamma_{A, i, j}(t)\right\rangle=\left\{\begin{array}{l}
1,(A, m, n)=(A, i, j) \\
0,(A, m, n) \neq(A, i, j),
\end{array}\right.
$$

where $\gamma_{A, i, j}(t)=\gamma(t-i T) O_{A, j}(t, u)$, then, based on the assumptions, the STLCS coefficients, $C_{A, m, n}$, with dual window $\gamma(t)$, are expressed as the following two forms:

$$
\begin{aligned}
C_{A, m, n} & =\int_{-\infty}^{+\infty} f(t) \gamma_{A, m, n}^{*}(t) d t \\
C_{A, m, n} & =\left\langle f(t), \gamma_{A, m, n}(t)\right\rangle
\end{aligned}
$$

According to Theorem 2 in [24], we have the following condition:

$$
\begin{aligned}
T_{0} \exp & \left\{\frac{j n T_{0}}{2}\left(n T_{0}-t\right) \frac{a}{b}\right\} \\
& \times \sum_{m} h(t-m T) \gamma^{*}\left(t^{\prime}-m T-n T_{0}\right)=\delta(n) .
\end{aligned}
$$


Equation (14) presents the orthogonal relationship between original window and dual one. Let $n=0$, according to properties of the Dirac function, we obtain

$$
T_{0} \sum_{n} h^{*}(t-n T) \gamma(t-n T)=1
$$

On the other hand, when $n \neq 0$, we have the following:

$$
\sum_{n} \gamma(t-n T) h^{*}\left(t-n T-m T_{0}\right)=0
$$

Equations (15) and (16) are derived for the subsequent computation process.

\section{Computation of Dual Window Function $\gamma(t)$}

4.1. Methodology. The object of this section is to find a Gaussian-like dual window which looks "similar" to the original one under the orthogonal condition. Given an original window function $h(t)$, its dual window $\gamma(t)$ of the basis $h_{A, m, n}(t)$ can be computed via minimizing energy of (20). We generalize the method used in [26] by solving its two prerequisites that need to be determined in deducing the process. One is the first terms of coefficients, choice of the period value $T_{0}$. In accordance with the choice in section 2 of [29], we adopt $T_{0}=b / \omega$. It denotes that the result depends on the determination of free parameter $\$ b \$$. The other one is that the derivation procedure should be based on specific form or corollary of orthogonal condition. We overcome it by Theorem 1 . And the shape of derived dual windows is affected by free parameters. Therefore, dual window can be modified through adjusting the parameters. The details will be given at the end of this section. The recursive approach is summarized as the following theorem.

Theorem 2. The minimum energy solution to the orthogonal condition of the STLCS is

$$
\gamma(t)=\sum_{m} a_{m}(t) h(t-m T)
$$

which denotes the relationship between original window function $h(t)$ and its dual window $\gamma(t)$, where $a_{m}(t)$ is a periodic function with period $T(m \in Z)$ and can be calculated via a recursive formula:

$$
\begin{aligned}
& a_{m}(t) \\
& =\frac{-\sum_{k=0}^{m-1} a_{k}(t) \sum_{n} h\left(t-n T-k T_{0}\right) h^{*}\left(t-n T-m T_{0}\right)}{\sum_{n}\left|h\left(t-n T-m T_{0}\right)\right|^{2}},
\end{aligned}
$$

where

$$
a_{0}(t)=\frac{\omega}{b \sum|h(t-n T)|^{2}} .
$$

Proof. Suppose $\left\{h_{A, m, n}\right\}$ constitutes a basis. According to (14), $\gamma(t)$ exists in $\left\{h_{A, m, n}\right\}$; that is to say $\gamma(t)$ can be represented in terms of $\left\{h_{A, m, n}\right\}$ :

$$
\begin{aligned}
& \gamma(t)=\sum_{k} \sum_{v} \alpha_{k v} \\
& \cdot \exp \left\{j\left[-\frac{1}{2}\left(\frac{a}{b} t^{2}+\frac{d}{b}(n \omega b)^{2}\right)+n t \omega\right]\right\} h(t \\
& -m T) .
\end{aligned}
$$

First, we compute the first term $a_{0}(t)$ and let

$$
\begin{aligned}
& a_{k}(t) \\
& \quad=\sum \alpha_{k v} \exp \left\{j\left[-\frac{1}{2}\left(\frac{a}{b} t^{2}+\frac{d}{b}(n \omega b)^{2}\right)+n t \omega\right]\right\}
\end{aligned}
$$

which is a periodic function. Thus, (20) can be rewritten as follows:

$$
\gamma(t)=\sum_{k} a_{k}(t) h(t-k T)
$$

This establishes (17). To calculate $a_{0}(t)$, let $k=0$ :

$$
\gamma(t)=a_{0}(t) h(t)+\varepsilon_{0}(t)
$$

where $\varepsilon_{0}$ is error between $\gamma(t)$ and $h(t)$; then we denote $\left\|\varepsilon_{0}(t)\right\|^{2}$ as follows:

$$
\begin{aligned}
\left\|\varepsilon_{0}\right\|^{2} & =\left\|\gamma(t)-a_{0}(t) h(t)\right\|^{2} \\
& =\int_{0}^{T_{0}} \sum_{n}\left|\gamma(t-n T)-a_{0}(t) h(t-n T)\right|^{2} d t \\
& =\int_{0}^{T_{0}} \sum_{n}\left|e_{0}(t, n)\right|^{2} d t,
\end{aligned}
$$

where $\|\cdot\|$ is norm, $e_{0}(t, n)=\gamma(t-n T)-a_{0}(t) h(t-n T)$, and, for $0 \leq t \leq T_{0}$, the minimizing $\left\|\varepsilon_{0}\right\|^{2}$ is equal to minimize $\Gamma$ as follows:

$$
\begin{aligned}
\Gamma= & \sum_{n}\left|e_{0}(t, n)\right|^{2} \\
= & \sum_{n}|\gamma(t-n T)|^{2}+\left|a_{0}(t)\right|^{2} \sum_{n} h(t-n T)^{2} \\
& -a_{0}(t) \sum_{n} h(t-n T) \gamma^{*}(t-n T) \\
& -a_{0}(t) \sum_{n} h^{*}(t-n T) \gamma(t-n T) .
\end{aligned}
$$

Then, suppose $a_{0}(t)=x+j y$. Let $\Theta=\partial / \partial x+j(\partial / \partial y)$; the minimized $\Gamma$ is achieved when

$$
\Theta_{\Gamma}=\frac{\partial \Gamma}{\partial x}+j \frac{\partial \Gamma}{\partial y}=0 .
$$


Therefore, we obtain

$$
\begin{aligned}
& 2 a_{0}(t) \sum_{n}|h(t-n T)|^{2}-2 \sum_{n} h^{*}(t-n T) \gamma(t-n T) \\
& \quad=0 .
\end{aligned}
$$

According to Theorem 1, we obtain

$$
T_{0} \sum_{n} h^{*}(t-n T) \gamma(t-n T)=1,
$$

where $T_{0}=b / \omega$, by substituting (28) into (27), we obtain

$$
a_{0}(t)=\frac{\omega}{b \sum_{n}|h(t-n T)|^{2}} .
$$

This establishes (19).

Second, the relationship between $a_{m}(t)$ and $a_{m-1}(t)$ can be derived using the same way in [26], and it is analogous to the derivation process of $a_{0}(t)$. To the last key steps,

$$
\begin{aligned}
& \sum_{n} \gamma(t-n T) h^{*}\left(t-n T-m T_{0}\right) \\
& \quad-\sum_{i=0}^{m-1} a_{i}(t) \sum_{n} h\left(t-n T-i T_{0}\right) h^{*}\left(t-n T-m T_{0}\right) \\
& \quad-a_{m}(t) \sum_{n}\left|h\left(t-n T-m T_{0}\right)\right|^{2}=0 .
\end{aligned}
$$

According to Theorem 1, we have

$$
\sum_{n} \gamma(t-n T) h^{*}\left(t-n T-m T_{0}\right)=0
$$

Equation (18) then follows.

Theorem 2 presents a computation approach for the dual $\gamma(t)$ with $h(t)$. From the process above, we can see that the main difference of computation process between the STLCS and the GS is determination of free parameter $b$.

4.2. Simulation. In this part, we choose a Gaussian window function, one of the most commonly used windows, as our original window function. Dual form of Gaussian window can be obtained via following steps:

(1) Choose, given Gaussian window $h(t)$, initial parameters including $T$ and $\omega b$.

(2) Compute $a_{m}(t)$ via $a_{0}(t), a_{1}(t), \ldots$ (It is not necessary to compute all the coefficients; in fact, the rest terms in $a_{m}(t)$ will approach zero even from the behind the third item.) $m T)$.

(3) Compute $\gamma(t)$ via Theorem 2: $\gamma(t)=\sum_{m} a_{m}(t) h(t-$

Example 3. Let $h(t)=(1 / \sqrt{2 \pi}) e^{-(1 / 2) t^{2}}$ (see Figure 2), and, then, we obtain

$$
\begin{aligned}
a_{m}(t)= & -\sum_{k=0}^{m-1} a_{k}(t) \exp \left[\frac{-\left((m-k)^{2} T_{0}^{2}\right)}{4}\right] \\
& \times \frac{\sum_{n} \exp \left[-\left(t-n T-(m+k) T_{0} / 2\right)^{2}\right]}{\sum_{n}\left|\exp \left(-\left(t-n T-m T_{0}\right)\right)\right|^{2}},
\end{aligned}
$$

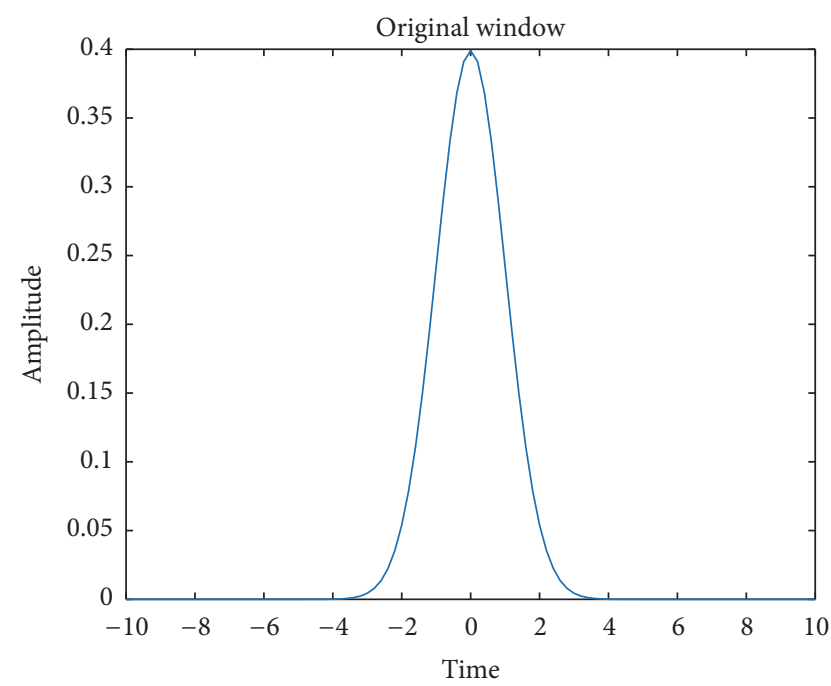

FIGURE 2: The given original Gaussian window function, $h(t)=$ $(1 / \sqrt{2 \pi}) e^{-(1 / 2) t^{2}}$.

where

$$
a_{0}(t)=\frac{2 \pi \omega}{b \sum_{n} \exp |-(t-n T)|^{2}} .
$$

Simplifying it, we obtain

$$
a_{m}(t)=-\sum_{k=0}^{m-1} a_{k}(t) \exp \left[\frac{-(m-k)^{2} T_{0}^{2}}{4}\right] .
$$

When $b=2 / \pi, \omega=1 / 2 \pi, T=0.9,(T \omega b \approx 0.0913)$, we can compute that $a_{0}(t)=2 \pi \omega / b=\pi / 2, a_{1}(t)=\pi / 2+$ $-\pi / 2 e, a_{2}(t)=\pi / 2+-\pi / 2 e+-\pi / 2 e^{4}$; the remaining terms in $a_{3}(t), a_{4}(t), \ldots, a_{m}(t)$ are very very small, and they can be ignored. So we obtain

$$
\begin{aligned}
\gamma(t) \approx & \frac{\pi}{2} h(t)+\left(\frac{\pi}{2}+\frac{-\pi}{2 e}\right) h(t-0.9) \\
& +\left(\frac{\pi}{2}+\frac{-\pi}{2 e}+\frac{\pi}{2 e^{4}}\right) h(t-1.8)+\cdots
\end{aligned}
$$

Other derived dual window $\gamma(t)$ with different parameters $b, \omega$, and $T$ can be calculated in a similar manner. On the one hand, when $b=2 / \pi, \omega=1 / 2 \pi$, original window and some dual windows with different $T$ are shown in Figure 3.

As a result, the shape of dual window $\gamma(t)$ with $T \omega b=$ $0.0101, T=0.1$ looks "most similar" to the original given window function $h(t)$. In fact, when $\omega b$ remains unchanged, the smaller $T$ is, the more similar $\gamma(t)$ and $h(t)$ look, which denotes that the value $T$ determines the size of the second lobe in dual window (see Figure 4). On the other hand, if we make $\omega$ and $T$ unchanged, the amplitude of the dual window can be affected by free parameter $b$ (see Figure 5). In any event, our object is to seek a dual window that shares shape characteristic of Gaussian window. Then, derived dual window not only can be used to substitute original window of the STLCS but also inherits good time-frequency properties 


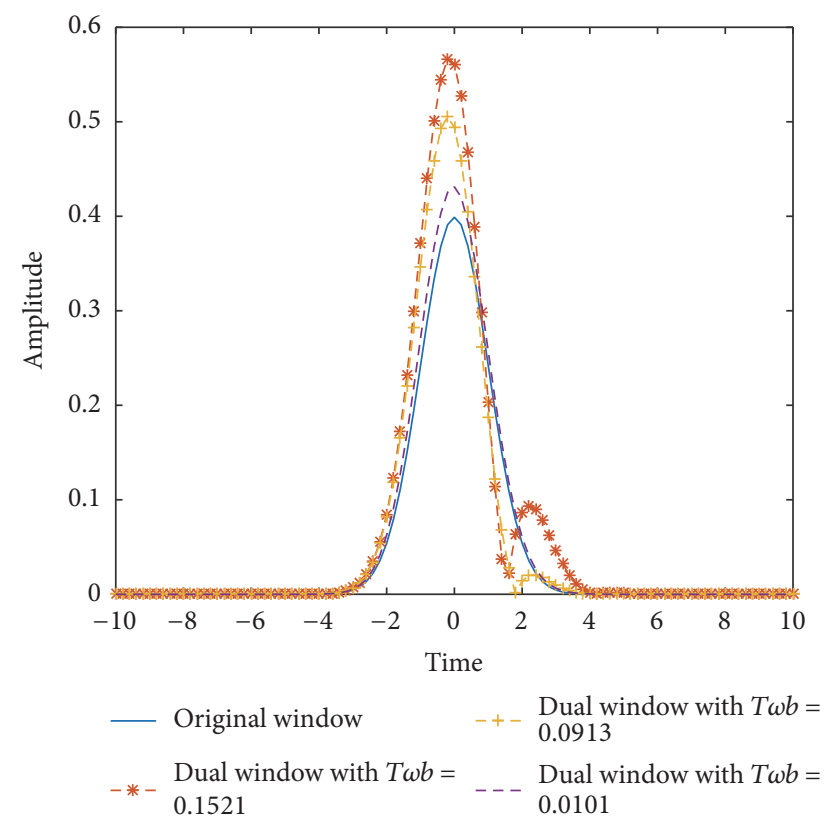

FIgURE 3: Comparison of original window and derived dual windows with different parameters. When $b=2 / \pi, \omega=1 / 2 \pi, T \omega b=$ $0.1521, T=1.5 ; T \omega b=0.0913, T=0.9 ; T \omega b=0.0101, T=0.1$.

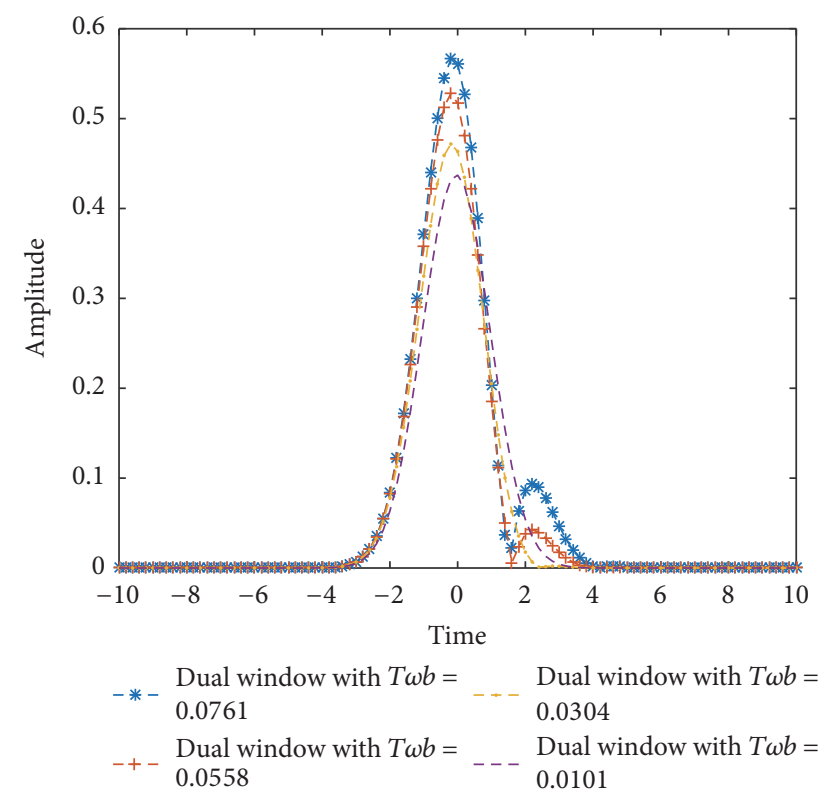

Figure 4: Comparison of derived dual windows with different parameters. When $b=1 / \pi, \omega=1 / 2 \pi, T \omega b=0.0761, T=1.5$; $T \omega b=0.0558, T=1.1 ; T \omega b=0.0304, T=0.6 ; T \omega b=0.0101$, $T=0.2$.

generated by original window. So, we are pleased to see the situation that $h(t)$ can be approximated by $\gamma(t)$ commendably.

4.3. Discussion. In this subsection, the second lobe and its influence are discussed. Simulation indicates that how free parameters affect the shape of derived dual windows. The

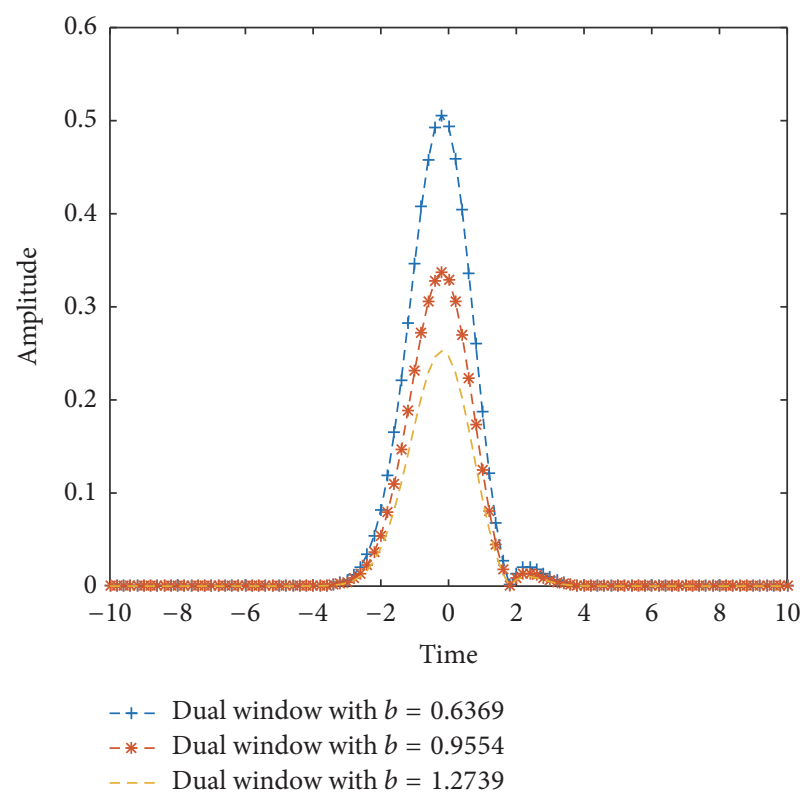

Figure 5: Comparison of derived dual windows with different $b$. When $T=0.9, \omega=1 / 2 \pi, T \omega b=0.0913, b=2 / \pi=0.6369$; $T \omega b=0.1369, b=3 / \pi=0.9554 ; T \omega b=0.1826, b=4 / \pi=1.2739$.

second lobe phenomena occur because of the computation expression of dual window. It can be eliminated by adjusting parameter $T$. More importantly, our object is to find a dual window which "looks like" Gaussian window shape as much as possible. It has its reasons. First, only the Gaussian window can achieve lower bound of uncertainty principle of STLCT, which implies that Gaussian window is an optimal window for the STLCT. Second, the larger second lobe means the dual window has a larger product value of $T \omega b$. According to the time-frequency theories of the STLCT, a larger value of $T \omega b$ can cause inverse STLCT to be more unstable than small value. Therefore, generally speaking, to achieve an optimal window and stable reconstruction, we usually avoid the occurrence of big second lobe by adjusting parameter.

The advantages of our work are as follows. We have proved that approach in [26] can be extended to the STLCS with two equivalent orthogonal conditions. Now we can compute the dual window if we have selected an appropriate initial term, which is a generalized form of the GS. It avoids the estimation of frame bounds and the Zak transform in [27]. More importantly, it asks weak orthogonal conditions to accomplish the derivation process. The whole computation is simple and concise.

The weakness of proposed method: although Gaussian window can be computed well, some other windows such as Triang window and Harming window cannot be calculated easily due to the complexity of the computational process. In particular, for the one-sided exponential window, it is not wise to compute its dual window by the approach. For example, consider the following window function:

$$
h(t)=0.5 e^{-0.5 t}
$$


We calculate $a_{0}(t)$ and $a_{m}(t)$ as follows:

$$
\begin{aligned}
& a_{0}(t)=\frac{\omega\left(1-e^{T}\right)}{0.5 b e^{-t}\left(1-e^{n T}\right)} \\
& a_{m}(t)=\frac{-0.5 \sum_{k=0}^{m-1} a_{k}(t) e^{-\left(t-(k / 2) T_{0}-(m / 2) T_{0}\right)}}{e^{-t+m T_{0}}} .
\end{aligned}
$$

The speed of $e^{-\left(t-(k / 2) T_{0}-(m / 2) T_{0}\right)} \rightarrow 0$ is too slow. This causes high computational complexity.

Potential applications of the STLCT: it is known that the classic GT has attracted much considerable research attention due to its time-frequency properties, ranging from signal and image processing to time series analysis domain, and so forth. For instance, in signal and image processing field, we can use the GT to deal with nonstationary signal in signal processing and also do the feature extraction in fault diagnosis and image processing field. As the generalization of the GT, the STLCT is a novel and flexible tool due to its free parameters. Another possible application area of the STLCT may be the efficient approximate representation in time series analysis. There was an indexing method for time sequences for processing similarity queries, in which FT was used to map time sequences to the frequency domain. However, first, the FT based indexing method cannot extract local information well due to its globe kernel function. The LCT maybe can handle this problem well by sliding window. Second, since the limitation of global transformation of the FT, this representation does not consider the time series local trends precisely, which can be extracted by the STLCT.

\section{Conclusion}

In this paper, we have developed two equivalent forms of orthogonal condition for the STLCS basis. A dual window solution to the nonorthogonal basis problem of the STLCT is presented. Then based on the condition, we can compute the dual window via recursive approach. The dual window computation is the prerequisite of the STLCS coefficients calculation. How free parameters affect the shape of dual window is studied. Finally, we investigate some advantages, weaknesses, and potential applications of the STLCT. This paper mainly focuses on its theoretical problem. The future work will be the study of applications of the STLCT.

\section{Conflicts of Interest}

The authors declare that there are no conflicts of interest regarding the publication of this paper.

\section{Acknowledgments}

This work was supported by the National Natural Science Foundation of China (61633005 and 61673076).

\section{References}

[1] L. Cohen, "Uncertainty principles of windowed wave functions," Optics Communications, vol. 179, no. 1, pp. 221-229, 2000.
[2] B. Deng, R. Tao, and Y. Wang, "Convolution theorems for the linear canonical transform and their applications," Science in China. Series F. Information Sciences, vol. 49, no. 5, pp. 592-603, 2006.

[3] Q. Feng and B.-Z. Li, "Convolution and correlation theorems for the two-dimensional linear canonical transform and its applications," IET Signal Processing, vol. 10, no. 2, pp. 125-132, 2016.

[4] H. Zhao, Q.-W. Ran, L.-Y. Tan, and J. Ma, "Reconstruction of bandlimited signals in linear canonical transform domain from finite nonuniformly spaced samples," IEEE Signal Processing Letters, vol. 16, no. 12, pp. 1047-1050, 2009.

[5] S.-C. Pei and S.-G. Huang, "Fast discrete linear canonical transform based on CM-CC-CM decomposition and FFT," IEEE Transactions on Signal Processing, vol. 64, no. 4, pp. 855866, 2016.

[6] X.-L. Fan, K. I. Kou, and M.-S. Liu, "Quaternion Wigner-Ville distribution associated with the linear canonical transforms," Signal Processing, vol. 130, pp. 129-141, 2017.

[7] K. K. Sharma and S. D. Joshi, "Uncertainty principle for real signals in the linear canonical transform domains," IEEE Transactions on Signal Processing, vol. 56, no. 7, part 1, pp. 26772683, 2008.

[8] J. Shi, X. Liu, and N. Zhang, "Generalized convolution and product theorems associated with linear canonical transform," Signal, Image and Video Processing, vol. 8, no. 5, pp. 967-974, 2014.

[9] Z. Zhang and M. Luo, "New integral transforms for generalizing the wigner distribution and ambiguity function," IEEE Signal Processing Letters, vol. 22, no. 4, pp. 460-464, 2015.

[10] H. Huo and W. Sun, "Sampling theorems and error estimates for random signals in the linear canonical transform domain," Signal Processing, vol. 111, pp. 31-38, 2015.

[11] Y.-L. Liu, K.-I. Kou, and I.-T. Ho, "New sampling formulae for non-bandlimited signals associated with linear canonical transform and nonlinear Fourier atoms," Signal Processing, vol. 90, no. 3, pp. 933-945, 2010.

[12] D. Wei and Y.-M. Li, "Sampling and series expansion for linear canonical transform," Signal, Image and Video Processing, vol. 8, no. 6, pp. 1095-1101, 2014.

[13] H. Zhao, R. Wang, and D. Song, "Recovery of bandlimited signals in linear canonical transform domain from noisy samples," Circuits, Systems, and Signal Processing, vol. 33, no. 6, pp. 19972008, 2014.

[14] Z.-C. Zhang, "New convolution structure for the linear canonical transform and its application in filter design," Optik, vol. 127, no. 13, pp. 5259-5263, 2016.

[15] R. G. Campos and J. Figueroa, "A fast algorithm for the linear canonical transform," Signal Processing, vol. 91, no. 6, pp. 14441447, 2011.

[16] S. Shinde, "Two channel paraunitary filter banks based on linear canonical transform," IEEE Transactions on Signal Processing, vol. 59, no. 2, pp. 832-836, 2011.

[17] J. Shi, X. Liu, L. He, M. Han, Q. Li, and N. Zhang, "Sampling and reconstruction in arbitrary measurement and approximation spaces associated with linear canonical transform," IEEE Transactions on Signal Processing, vol. 64, no. 24, pp. 6379-6391, 2016.

[18] Q. Zhang, "Zak transform and uncertainty principles associated with the linear canonical transform," IET Signal Processing, vol. 10, no. 7, pp. 791-797, 2016. 
[19] J. Shi, X. Liu, and N. Zhang, "On uncertainty principles for linear canonical transform of complex signals via operator methods," Signal, Image and Video Processing, vol. 8, no. 1, pp. 85-93, 2014.

[20] K.-I. Kou and R.-H. Xu, "Windowed linear canonical transform and its applications," Signal Processing, vol. 92, no. 1, pp. 179-188, 2012.

[21] K.-I. Kou, R.-H. Xu, and Y.-H. Zhang, "Paley-Wiener theorems and uncertainty principles for the windowed linear canonical transform," Mathematical Methods in the Applied Sciences, vol. 35, no. 17, pp. 2122-2132, 2012.

[22] Z.-C. Zhang, "Sampling theorem for the short-time linear canonical transform and its applications," Signal Processing, vol. 113, pp. 138-146, 2015.

[23] X. Guanlei, W. Xiaotong, and X. Xiaogang, "Uncertainty inequalities for linear canonical transform," IET Signal Processing, vol. 3, no. 5, pp. 392-402, 2009.

[24] L. Huang, K. Zhang, Y. Chai, and S. Xu, "Uncertainty principle and orthogonal condition for the short-time linear canonical transform," Signal, Image and Video Processing, vol. 10, no. 6, pp. 1177-1181, 2016.

[25] M. J. Bastiaans, "Gabor's Expansion of a Signal into Gaussian Elementary Signals," Proceedings of the IEEE, vol. 68, no. 4, pp. 538-539, 1980.

[26] Y. Lu and J. M. Morris, "Fast computation of gabor functions," IEEE Signal Processing Letters, vol. 3, no. 3, pp. 75-78, 1996.

[27] M. Zibulski and Y. Y. Zeevi, "Oversampling in the Gabor Scheme," IEEE Transactions on Signal Processing, vol. 41, no. 8, pp. 2679-2687, 1993.

[28] S. Qian and D. Chen, "Discrete Gabor Transform," IEEE Transactions on Signal Processing, vol. 41, no. 7, pp. 2429-2438, 1993.

[29] J. Zhao, R. Tao, and Y. Wang, "Sampling rate conversion for linear canonical transform," Signal Processing, vol. 88, no. 11, pp. 2825-2832, 2008. 


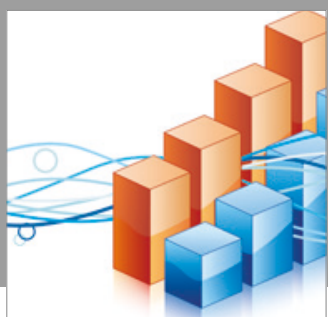

Advances in

Operations Research

vatersals

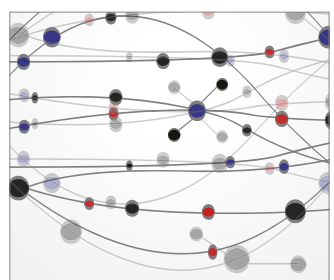

\section{The Scientific} World Journal
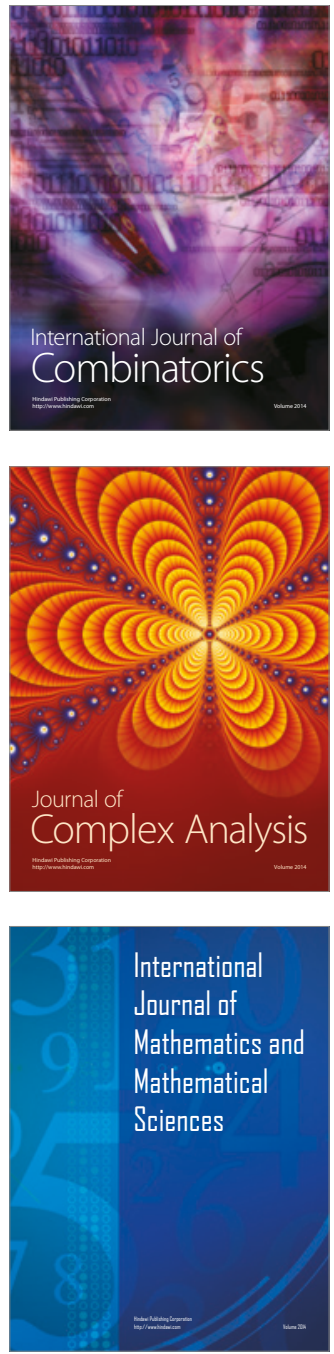
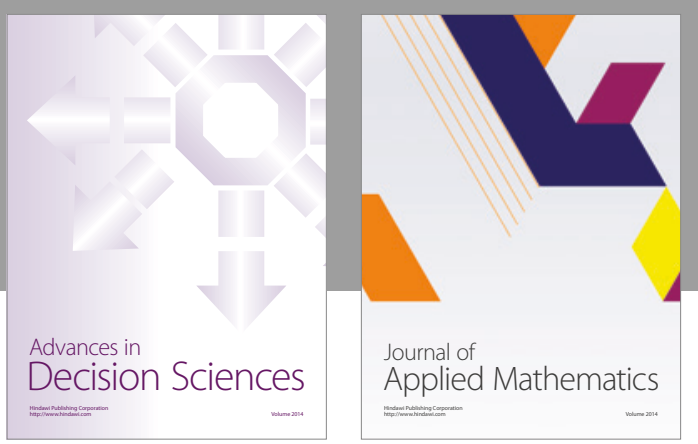

Algebra

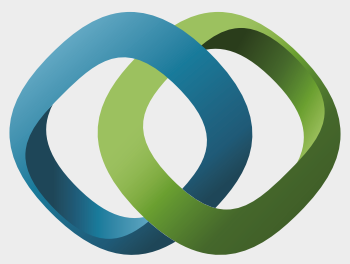

\section{Hindawi}

Submit your manuscripts at

https://www.hindawi.com
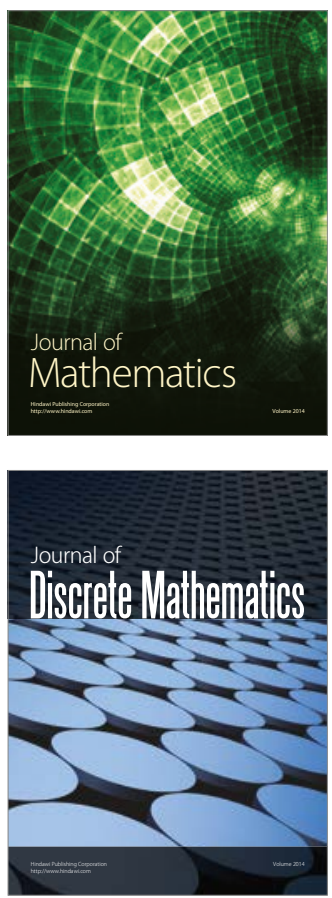

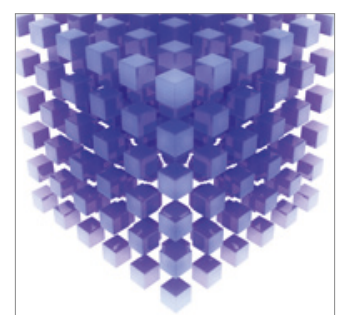

Mathematical Problems in Engineering
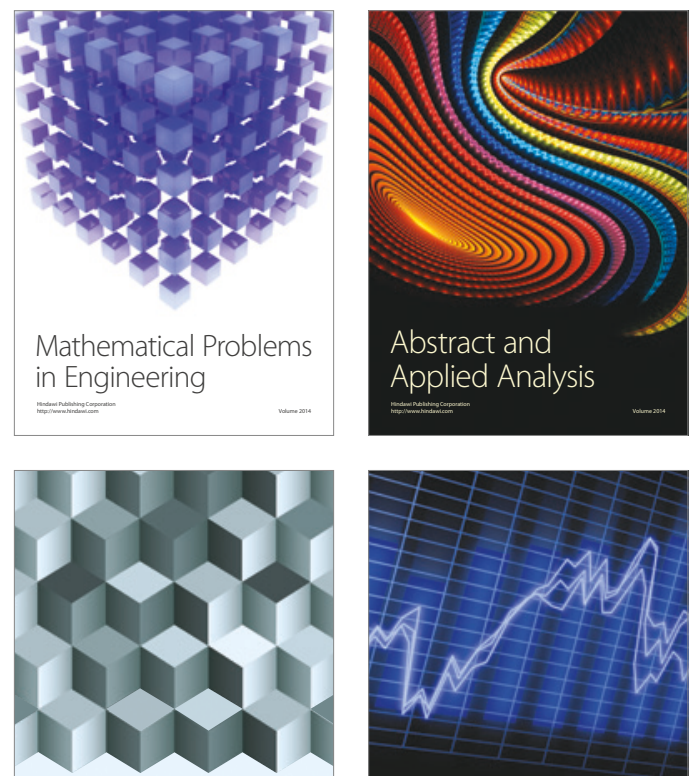

Journal of

Function Spaces

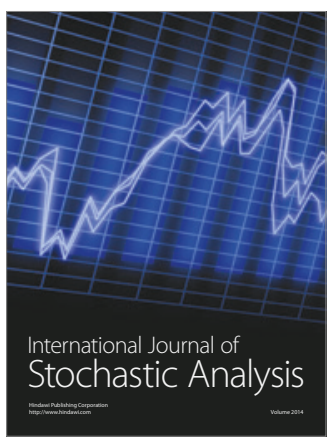

Probability and Statistics
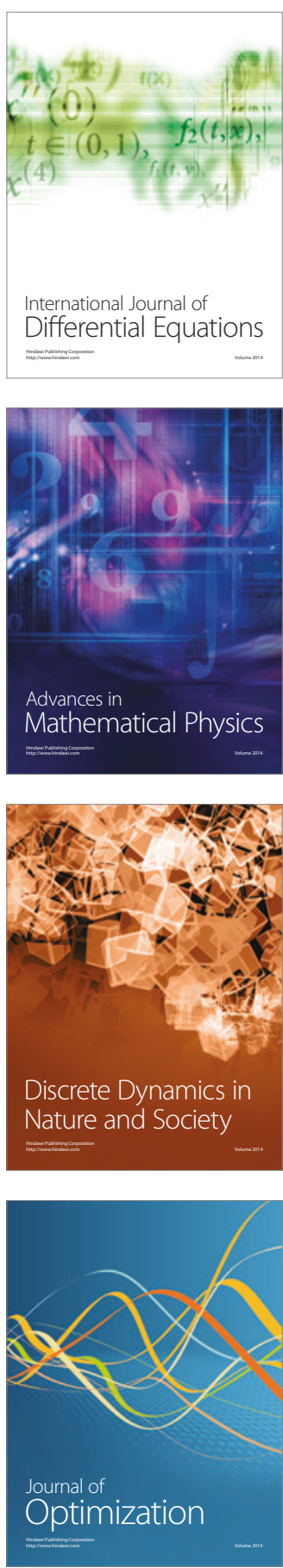